\title{
Süße Versuchung: De-novo-Wege zu ungewöhnlichen Zuckern
}

\author{
Christian Brand, Daniel B. Werz
}

Statt natürlich vorkommende Zucker zu modifizieren, setzen Chemiker zunehmend darauf,

Kohlenhydratgerüste von Grund auf neu zu synthetisieren. Dabei lassen sie sich von natürlichen

Vorbildern, den Aldolasen, inspirieren.

Die Rolle der Kohlenhydrate in der Natur ist wegen ihrer immensen Komplexität noch immer nicht vollständig verstanden. Einfache Polymere wie Cellulose als Gerüstsubstanz in Pflanzen oder Glykogen als Energiespeicher im menschlichen und tierischen Organismus sind besser untersucht als Zucker, die biologische Information kodieren. Dennoch beruhen sehr viele Zell-Zell-Erkennungsvorgänge auf Zucker-Zucker-Interaktionen. Zum Beispiel sind den menschlichen Blutgruppen im AB0-System jeweils spezifische Kohlenhydrat-Antigene zugeordnet und Bakterien nutzen Zuckerstrukturen, um an ihre Wirte anzudocken. Gerade Bakterien bedienen sich dafür teils sehr komplexer Monosaccharide, deren chemische Synthese häufig schwierig ist. Früher modifizierten Chemiker dazu die im Chiral Pool ubiquitären $\mathrm{Zu}$ cker wie Glucose, Galactose und Mannose so, dass das gewünschte Funktionalitätsmuster entstand. Meist war dies jedoch nur mit aufwendigen Schutzgruppenstrategien möglich. Erst in jüngerer Zeit haben De-novo-Synthesen, also Ansätze, die das Kohlenhydratgerüst ganz oder auch nur teilweise neu aufbauen, an Bedeutung gewonnen.

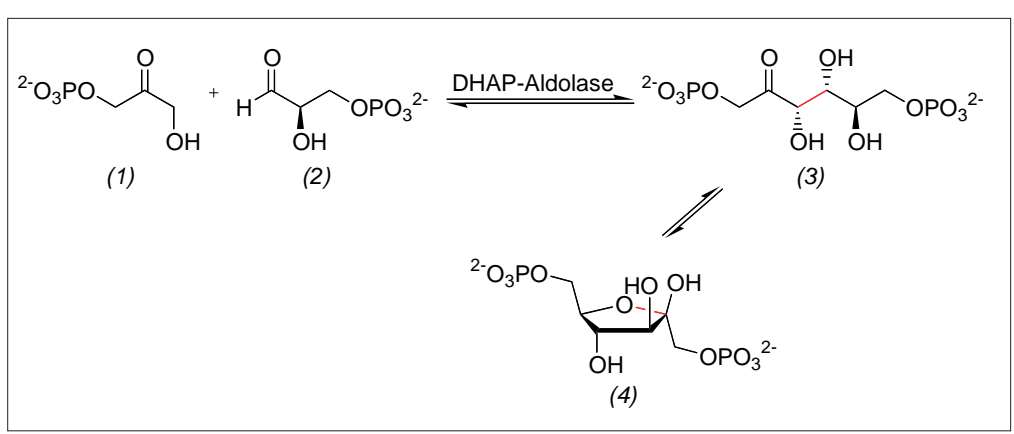

Abb. 1. Enzymkatalysierte De-novo-Synthese von Kohlenhydraten.

\section{Asymmetrische Aldolreaktionen}

- Wertvolle Beispiele für die De-novo-Synthese von Zuckern finden sich in der Natur: Um Hexosen aufzubauen, nutzt die Natur die $\mathrm{C}_{3}$-Bausteine Dihydroxyacetonphosphat (DHAP) (1) und Glycerinaldehyd-3-phosphat (2) (Abbildung 1). ${ }^{1)}$ Als Produkt dieser enzymatisch katalysierten diastereoselektiven Aldolreaktion entsteht D-Fructose-1,6-diphosphat (3), das der Natur zur Gewinnung von Fructose, Glucose und Mannose dient. Enzymatisch vermittelte Substitutionsreaktionen, reduktiv-oxidative Funktionalisierungen und Desoxygenierungen erlauben danach eine entsprechende Diversifizierung der zugrundeliegenden Strukturen.

Inspiriert durch das natürliche Vorbild, die Aldolasen, haben Chemiker ausgehend von $\beta$-Hydroxycar- bonylverbindungen eine Reihe organokatalytischer Verfahren entwickelt, um Kohlenhydrate oder Kohlenhydratvorläufer aufzubauen. Besonders bemerkenswert ist dabei die von MacMillan et al. 2004 beschriebene asymmetrische Zwei-Stufen-Synthese von Aldohexosen (Abbildung 2, S. 526). ${ }^{2,3)}$ Im ersten Schritt wird dazu der silylgeschützte Glycolaldehyd (5), ein $C_{2}$-Baustein, in Gegenwart von L-Prolin mit einer Ausbeute von $92 \%$ zu dem entsprechenden Aldolprodukt (6) umgesetzt. Dabei entstehen zwei neue Stereozentren mit einer Diastereoselektivität von 4:1 zugunsten des AntiProdukts und einem exzellenten Enantiomerenüberschuss von $95 \%$. Auch andere elektronenreiche $\mathrm{O}$-alkylierte Glycolaldehyde erzielen hinsichtlich Ausbeute und Enantiomerenüberschuss gute Ergebnisse, elektronenziehende Substituenten wie Acetatgruppen unterdrücken 


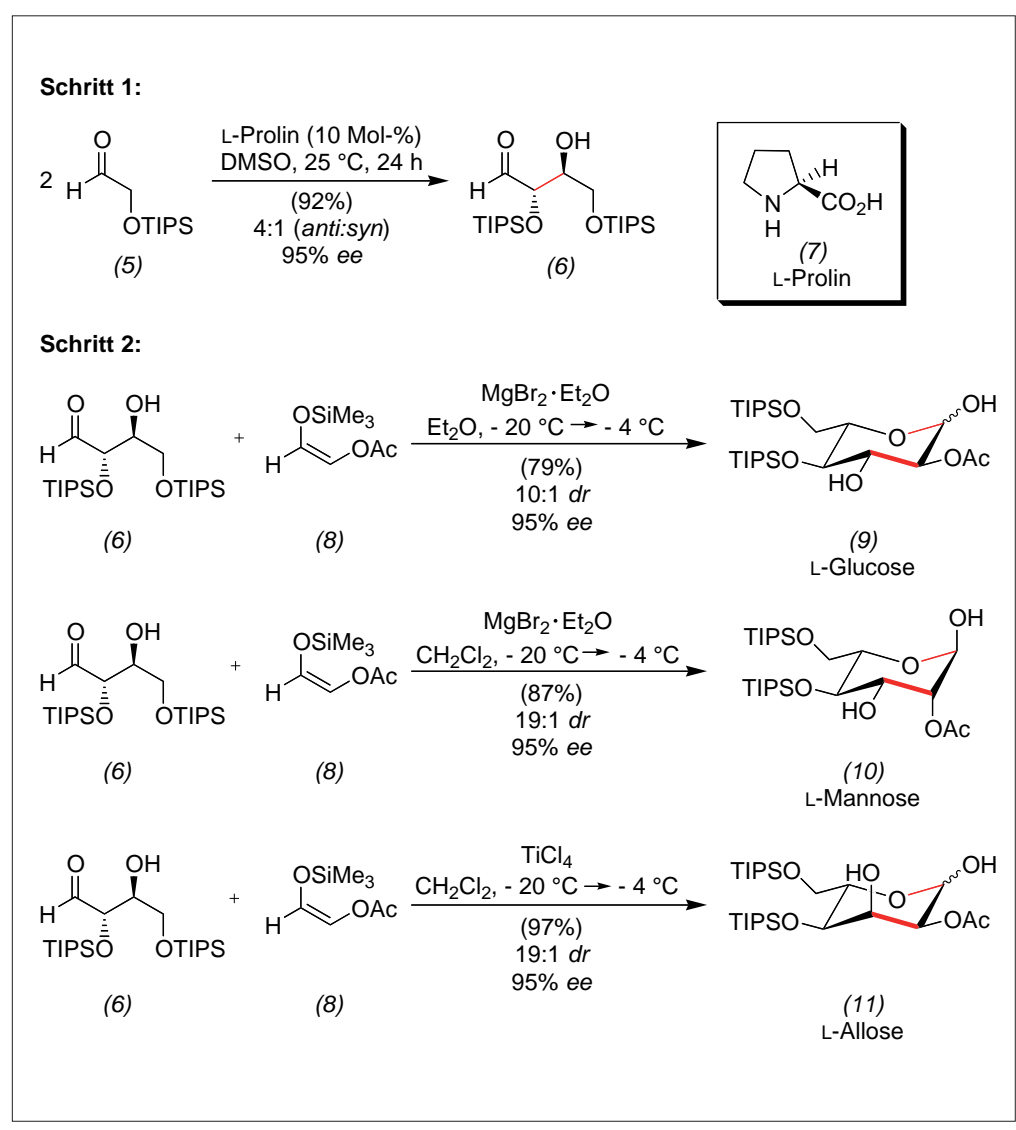

Abb. 2. Schritt 1: enantioselektive Dimerisierung des silylierten a-Hydroxyaldehyds (5). Schritt 2: Lewis-Säure-vermittelte Mukaiyama-Aldol-Reaktion zum Aufbau des $C_{6}$-Fragments nach MacMillan et al.

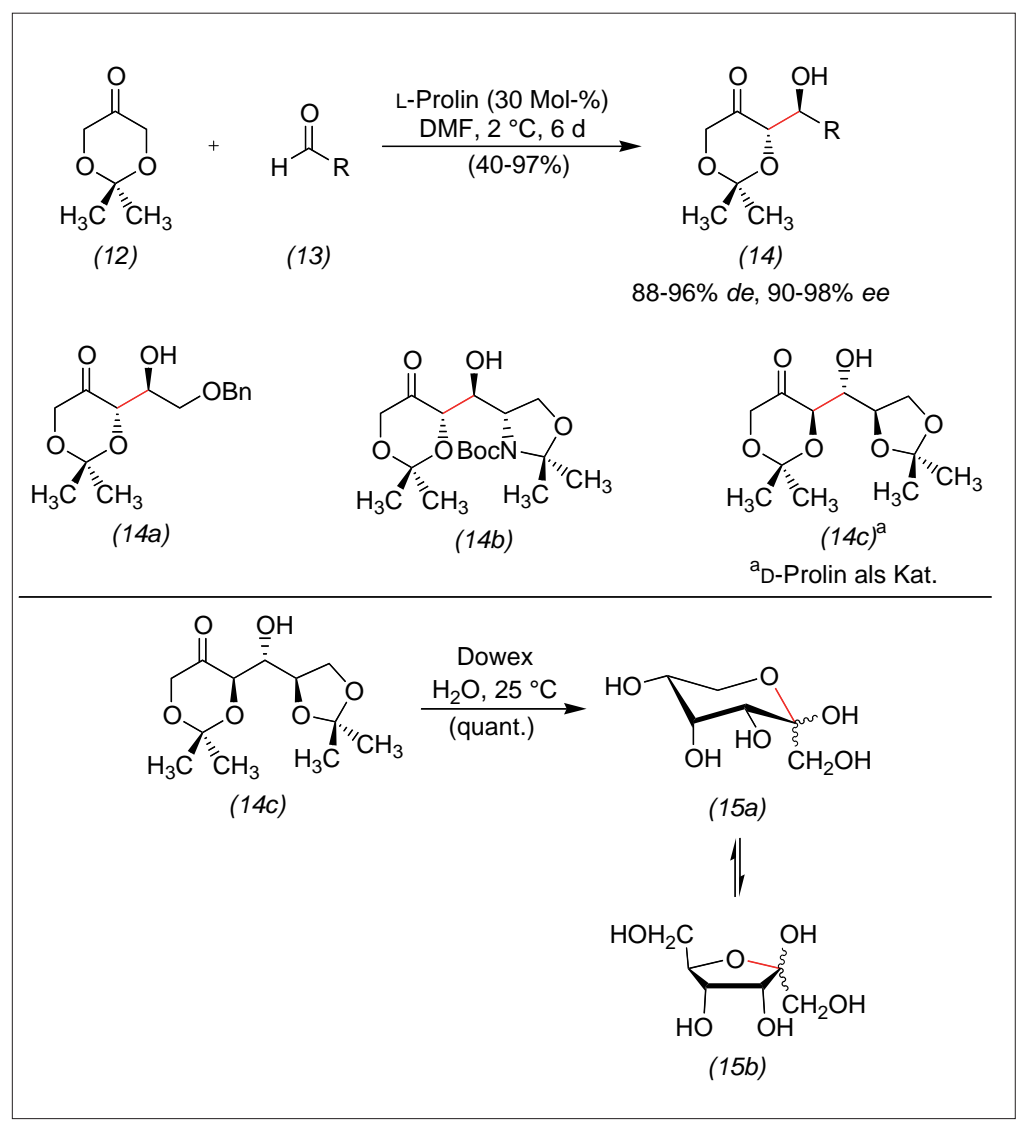

Abb. 3. L- und D-Prolin-katalysierte asymmetrische Aldolreaktion von Dioxanon mit Aldehyden nach Enders et al. (oben); Erzeugung der entschützten Zucker (unten). dagegen die Aldoladdition. Interessanterweise bleibt die Reaktion des gebildeten Aldolprodukts (6) mit einem weiteren Äquivalent des silylgeschützten Glycolaldehyds (5) aus.

Der zweite Schritt ist eine Aldolreaktion unter Mukaiyama-Bedingungen mit dem Silylenolether (8). Dabei spielen die Reaktionsbedingungen eine entscheidende Rolle, um die zum Teil geschützten Kohlenhydrate (9-11) selektiv zu erzeugen. Magnesiumbromid in Diethylether als Lewis-Säure liefert das entsprechende Glucose-Derivat (9), während dieselbe Lewis-Säure in Dichlormethan die Konfiguration an C-2 des Sechsrings hin zur Mannose (10) verändert. Mit Titan(IV)chlorid entsteht die entsprechende Allose (11). Insgesamt bauten MacMillan et al. so ausgehend von achiralen Startmaterialien mit Prolin als chiralem Organokatalysator vier Stereozentren gezielt auf. In allen Fällen waren die Diastereoselektivitäten bei der Mukaiyama-Aldolreaktion hoch (über 10:1).

Enders et al. bauten Ketohexosen durch prolinkatalysierte Aldoladdition (Abbildung 3) auf. ${ }^{4)}$ Im Jahr 2005 berichteten sie über die erfolgreiche Entwicklung der ersten diastereound enantioselektiven organokatalytischen Aldolreaktion mit 2,2-Dimethyl-1,3-dioxan-5-on (Dioxanon) (12), einem $\mathrm{C}_{3}$-Baustein - ähnlich wie die Natur ihn nutzt. Mit geeigneten $\mathrm{C}_{2^{-}}$und $\mathrm{C}_{3}$-Aldehydkomponenten ist ein direkter Aufbau von selektiv geschützten Kohlenhydraten in einem Schritt möglich. Hervorzuheben sind die hohen Diastereomerenüberschüsse von $88-96 \%$ und die bemerkenswerten Enantiomerenüberschüsse von $90-98 \%$. Ähnlich wie beim Ansatz von MacMillan ist eine Anti-Selektivität beim Aufbau der zwei neu gebildeten Stereozentren zu beobachten. Die besten Ergebnisse liefert 30 Mol-\% Prolin in Dimethylformamid bei $2{ }^{\circ} \mathrm{C}$ und einer langen Reaktionszeit von sechs Tagen. Die Abspaltung der Isopropyliden-Schutzgruppen durch saures Dowex-Harz liefert die freien Zucker. Die offenkettige Ketohexose D-Psicose (14c) 
schließt sich danach zu einer Pyranose (15a) und einer Furanose $(15 b)$, die miteinander im Gleichgewicht stehen.

Um dem Konzept der Natur zum Aufbau von Kohlenhydraten durch DHAP näher zu kommen, entwickelten Mahrwald et al. 2007 ein zweistufiges Konzept für ungeschützte Furanosen (Abbildung 4). ${ }^{5}$ Die intermolekulare direkte AldolAddition des Ketons (16) mit Aldehyd (17) ermöglicht mit der sterisch anspruchsvollen Base DBU einen Zugang zu den offenkettigen an C-5 geschützten Hexoketosen (18) und (19). Diese entstehen in einem Diastereomerenverhältnis von $1: 1$. Die Reaktion lässt sich bei Raumtemperatur und ohne Lösungsmittel durchführen. Im Gegensatz zu den zuvor beschriebenen Aldol-Additionen mit L-Prolin beobachtet man in diesem ausschließlich substratkon- trollierten Fall eine Syn-Selektivität der beiden neu gebildeten Stereozentren. Nach Entfernung der Silyletherschutzgruppe mit Tetrabutylammoniumfluorid (TBAF) und der folgenden In-situ-Cyclisierung entstehen die Ketofuranosen (20) und (21) mit einer Ausbeute von 51\% über zwei Stufen.

Wenn als $C_{3}$-Aldehydkomponente isopropylidengeschützter (R)Glycerinaldehyd (22) dient, gelingt mit dem chiralen Alkaloid (-)-Cinchonin (23) (30 Mol-\%) mit Dihydroxyaceton eine stereoselektive Aldol-Addition (Abbildung 5, S. 528). Sie liefert in enantiomerenreiner Form mit $71 \%$ Ausbeute D-Fructose $(24)^{5)}$

Aldol-Additionen erlauben auch den Aufbau von Zuckern mit quartären Stereozentren. Wie Mahrwald et al. 2009 zeigten, ist eine asymmetrische Aldol-Reaktion von enolisier-

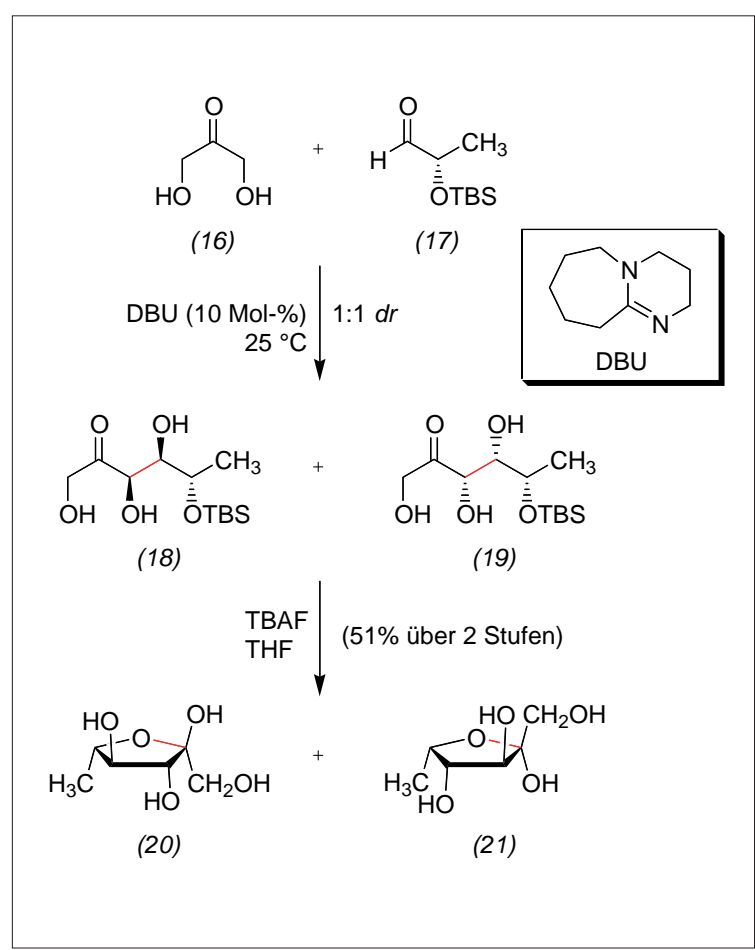

Abb. 4. De-novo-Synthese von L-Rhamnulofuranose (20) und 6-Desoxy-L-sorbofuranose (21) nach Mahrwald et al.

\section{HORIBA}

Scientific

www.horiba.com/de/scientific
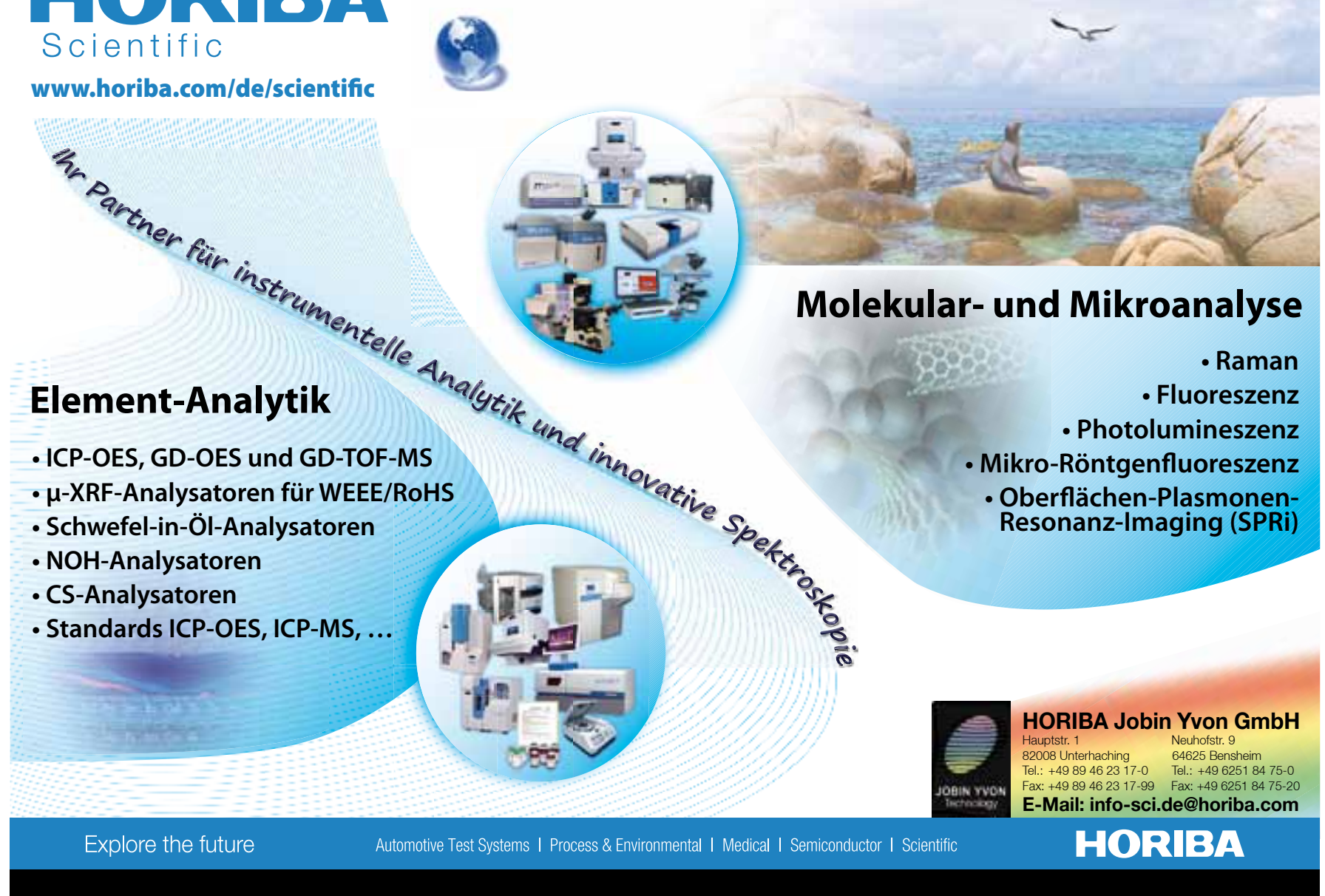


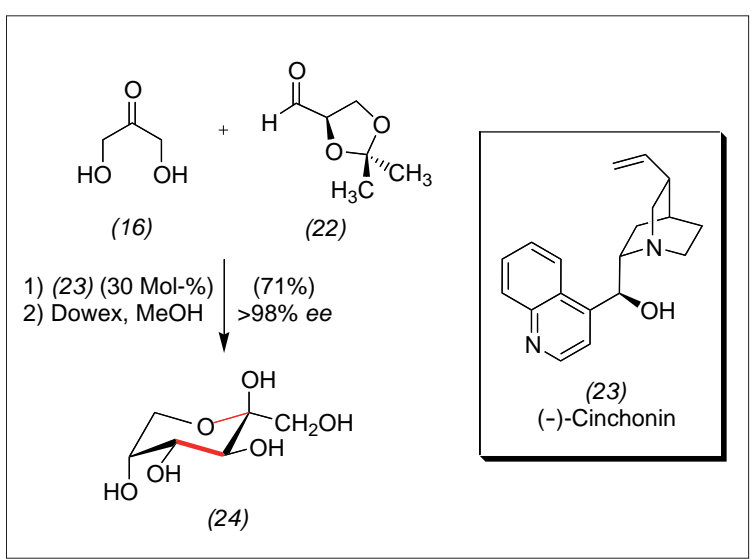

Abb. 5. Cinchoninkatalysierte Aldoladdition von Dihydroxyaceton (16) und (R)-Gycerinaldehyd (22) zu D-Fructose (24) nach Mahrwald et al. baren Aldehyden hierzu das Mittel der Wahl (Abbildung 6, S. 528). ${ }^{6)}$ So gelang die stereoselektive Homodimerisierung des acetonidgeschützten (R)-Glycerinaldehyds (22) in Anwesenheit katalytischer Mengen der Aminosäure L-Histidin. So entsteht in $82 \%$ Ausbeute über zwei Stufen ein Zugang zu nahezu enantiomerenreiner 2-HydroxymethylD-lyxose (25). Analog liefert die D-Histidin-katalysierte Dimerisierung des benzylgeschützten $\mathrm{Hy}$ droxypropanals (26) gefolgt von einer Hydrogenolyse zur Abspaltung

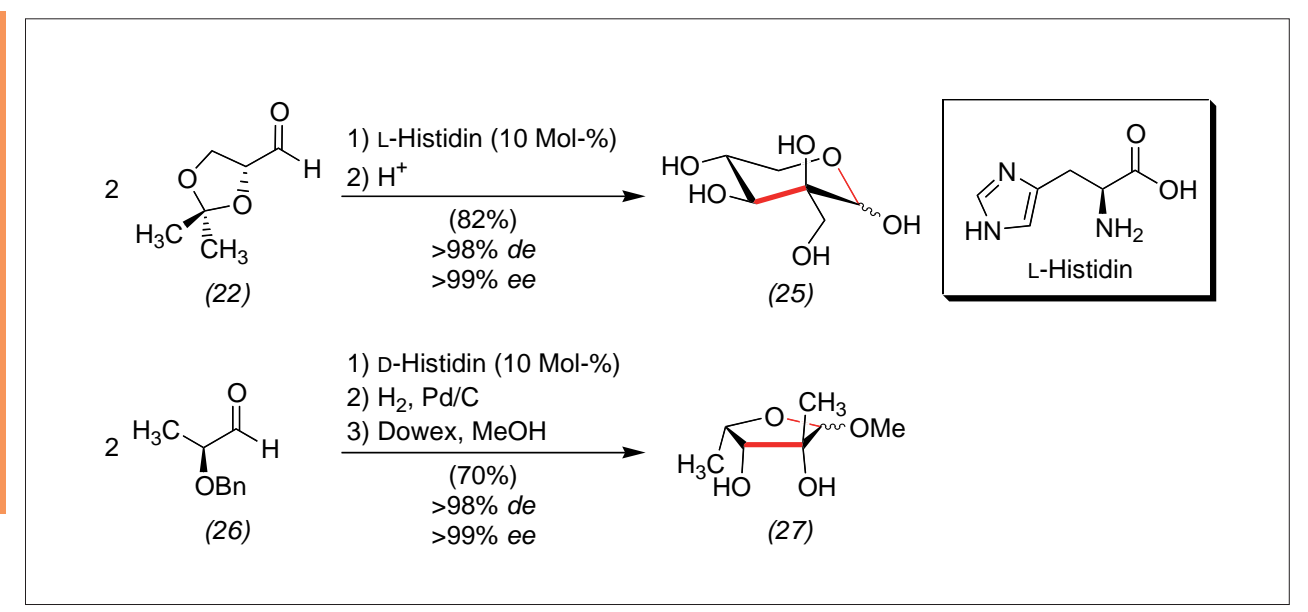

Abb. 6. Asymmetrische histidinkatalysierte De-novo-Synthese zum Aufbau von Kohlenhydraten mit quartären Stereozentren.

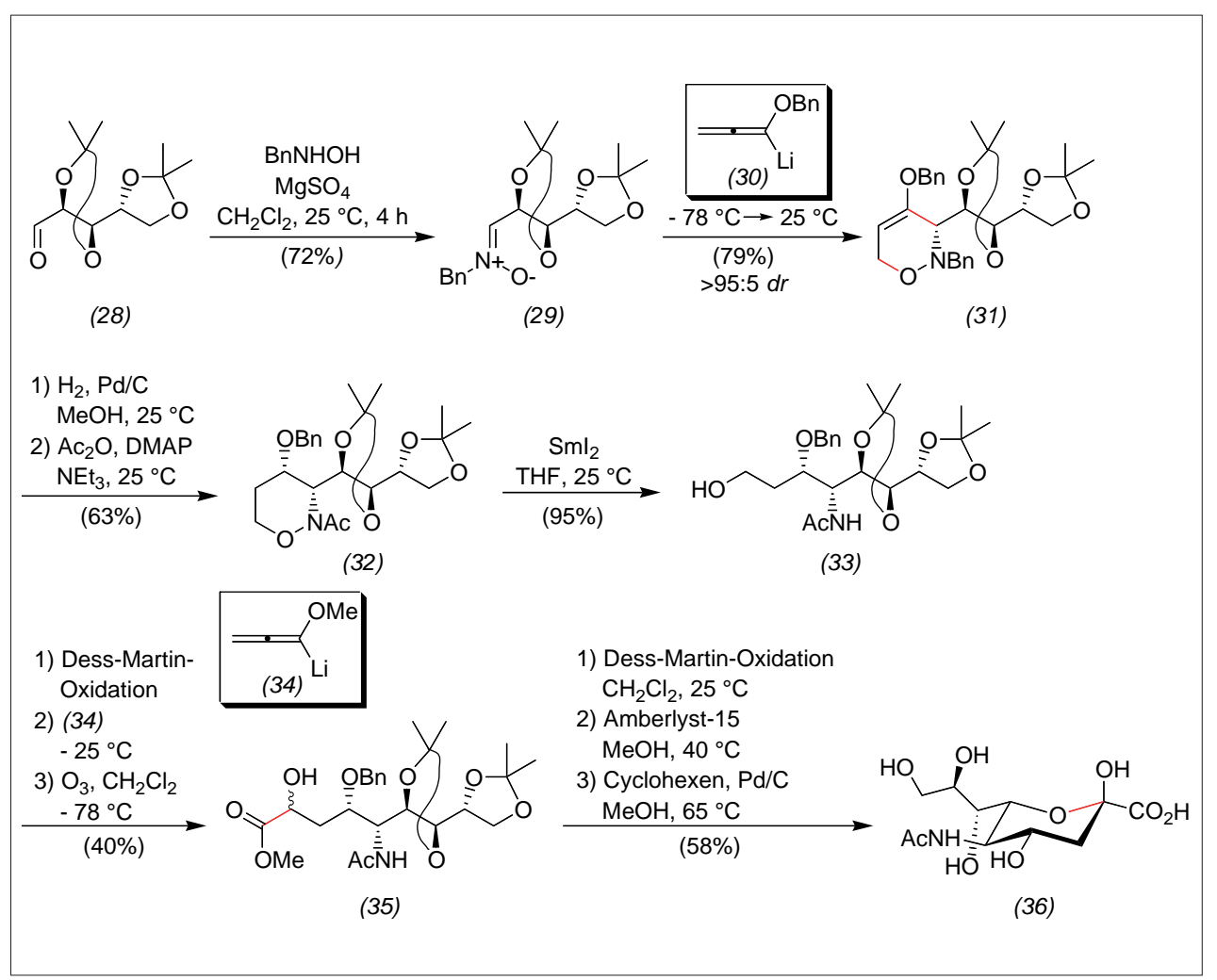

Abb. 7. De-novo-Synthese von Sialinsäure (36) nach Reißig et al. der Benzylschutzgruppe die 5-Desoxy-2-methyl-L-lyxose (27) als einziges Stereoisomer mit sehr guten Ausbeuten von $70 \%$.

\section{Ansätze mit Allenen und über Palladiumkatalyse}

- Auch wenn der Siegeszug der Organokatalyse im letzten Jahrzehnt erstaunliche Synthesen von ungewöhnlichen Monosacchariden ermöglicht hat, trugen auch andere Gebiete der organischen Synthesechemie zu Fortschritten in der Denovo-Synthese bei. Mit dem Schlüsselschritt einer stereoselektiven [3+3]-Cyclisierung eines Nitrons mit einem lithiierten elektronenreichen Allen synthetisierten Reißig et al. eine Reihe von komplexen Monosacchariden und Monosaccharid-Derivaten. ${ }^{7,8)}$ In der Sialinsäuresynthese aus Abbildung 7 reagiert das von geschützter D-Arabinose abgeleitete Nitron (29) als $C_{5}$-Baustein mit dem lithiierten Allen (30) als $C_{3}$-Baustein. Im entstehenden 1,2-Oxazinderivat (31) sind die Positionen der funktionellen Gruppen in der späteren Sialinsäure (36) bereits vorfixiert. Die Carboxylgruppe des Zuckers wird ebenfalls über ein lithiiertes Allen eingebracht, das nach einer Ozonolyse als $\mathrm{C}_{1}$-Baustein fungiert und das Methylcarboxylat in (35) liefert. Nach Oxidation der freien OH-Gruppe zum Keton und der Abspaltung der Isopropyliden- und Benzylschutzgruppen bildet sich der Sechsring der Sialinsäure (36).

Ausgehend von 2-Acetylfuran gelangen O'Doherty beeindruckende Synthesen von Monosacchariden. ${ }^{9}$ Ein Beispiel zeigt Abbildung 8: Eine Noyori-Reduktion von (37) ergibt zunächst mit hohem Enantiomerenüberschuss den Furfurylalkohol (38). Eine Oxidation des Furansystems mit N-Bromsuccinimid (NBS) führt in Anwesenheit von $\mathrm{H}_{2} \mathrm{O}$ über eine Achmatowicz-Umlagerung zum Hydroxypyranon. Die über das entsprechende Anhydrid eingeführte Boc-Schutzgruppe wird in einer palladiumkatalysierten allylischen Sub- 
stitution durch eine Benzylschutzgruppe ersetzt. Die verbleibende Doppelbindung lässt sich diastereoselektiv in Anti-Stellung zur schon vorhandenen, geschützten Hydroxylgruppe epoxidieren. Mit hoher Diastereoselektivität erfolgt die anschließende Luche-Reduktion der Ketogruppe zur äquatorialen $\mathrm{OH}$ Gruppe. Das so erhaltene Epoxid eröffnet sowohl die Möglichkeit zum Aufbau des Altrose-Gerüsts (43) als auch des desoxygenierten Monosaccharids (44). Entsprechende Funktionalisierungen der Enone sind auch an Oligomeren möglich, die über palladiumkatalysierte allylische Glycosylierungen aufgebaut wurden. So ließen sich bereits größere biologisch aktive Oligosaccharide synthetisieren. ${ }^{10)}$

\section{Zusammenfassung und Ausblick}

- Die rasante Entwicklung der Organokatalyse im letzten Jahrzehnt hat einfache Wege zum Aufbau komplexer Zucker erschlossen. Inspiriert durch den biosynthetischen Zugang zu Hexosen ließen sich so einige ungewöhnliche Monosaccharide aufbauen. Die neu entwickelten Methoden machen zudem isotopenmarkierte Verbindungen zugänglich und sollten daher für die Biochemie von großem Wert sein. Für Kohlenhydratchemiker, die sich mit dem Aufbau komplexer Glycane beschäftigen, sind Monomere mit einem Schutzgruppenmuster aus perma-nenten und temporären Schutzgruppen sowie geeigneter anomerer Abgangsgruppe von großer Bedeutung. Erste Erfolge gibt es auch bei der De-novoSynthese derartiger Bausteine. ${ }^{11,12)}$ Die Vielfalt bakterieller Oligosaccha-

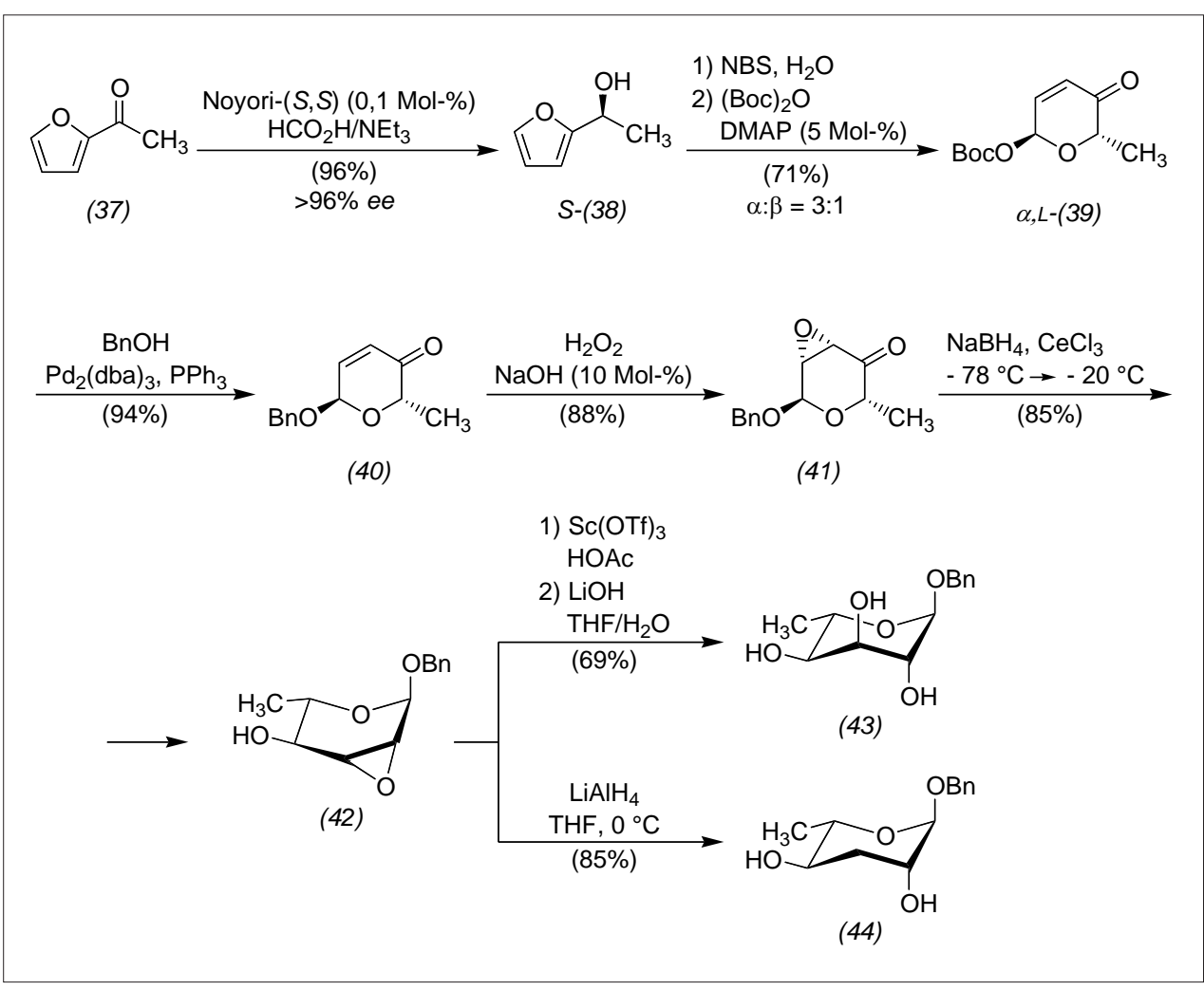

Abb. 8. Synthese von Desoxyaltropyranosen nach O'Doherty et al.

ride mit ihren ungewöhnlichen Bausteinen wird zu weiteren neuartigen De-novo-Synthesen anregen.

$\overline{\text { Blickpunkt Synthese will zur Beschäftigung }}$ mit neuen synthetischen Verfahren oder eleganten und wichtigen Synthesen aus der Literatur anregen. Die Rubrik betreut in diesem Jahr Daniel B. Werz. Er ist Emmy-NoetherNachwuchsgruppenleiter an der Universität Göttingen. Bei diesem Beitrag unterstützte ihn Christian Brand, Doktorand an der Universität Göttingen. dwerz@gwdg.de

Literatur

1) T.D. Machajewski, C.-H.Wong, Angew. Chem. 2000, 112, 1406-1430.

2) A. B. Northrup, D. W. C. MacMillan, Science 2004, 305, 1752-1755.

3) A. B. Northrup, I. K. Mangion, F. Hettche, D. W. C. MacMillan, Angew. Chem. 2004, 116, 2204-2206.
4) D. Enders, C. Grondal, Angew. Chem. 2005, 117, 1235-1238.

5) M. Markert, M. Mulzer, B. Schetter, R. Mahrwald, J. Am. Chem. Soc. 2007, 129, 7258-7259.

6) M. Markert, U. Scheffler, R. Mahrwald, J. Am. Chem. Soc. 2009, 131, 16642-16643.

7) B. Bressel, H.-U. Reissig, Org. Lett. 2009, 11, 527-530.

8) F. Pfrengle, D. Lentz, H.-U. Reissig, Angew. Chem. 2009, 121, 3211-3215.

9) M. Shan, Y. Xing, G. A. O'Doherty, J. Org. Chem. 2009, 74, 5961-5966.

10) H. Guo, G. A. O'Doherty, Angew. Chem. 2007, 119, 5298-5300.

11) M. S. M. Timmer, A. Adibekian, P. H. Seeberger, Angew. Chem. 2005, 117, 7777-7780.

12) A. Adibekian, M. S. M. Timmer, P. Stallforth, J. van Rijn, D. B. Werz, P. H. Seeberger, Chem. Comm. 2008, 3549-3551.

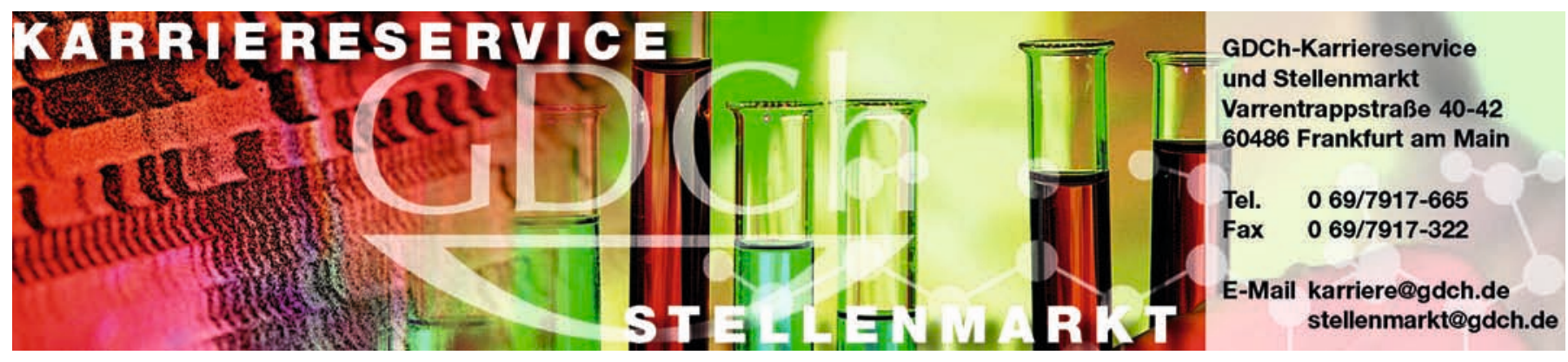

